\title{
Presentación. El espacio urbano en el año 2020. Reflexiones en el marco de la contingencia sanitaria
}

\author{
Eduardo Sousa-González ${ }^{1}$
}

$P^{2}$ on este número publicado en el mes de septiembre, CONTEXTO. Revista de la Facultad de Arquitectura de la Universidad Autónoma de Nuevo León, cierra sus publicaciones en este año del 2020; primeramente, con una noticia muy importante para la Revista y seguramente para todos los interesados en la publicación, ya que CONTEXTO fue evaluada y aceptada en el índice de SCOPUS y a la brevedad se terminará el proceso para completar su indexación; por otro lado, el año 2020, visiblemente se ha caracterizado por una creciente e incontrolable contingencia sanitaria y pandémica mundial.

Un año catastrófico sin paralelismo reciente, que seguramente será recordado en la esfera global, no solo por lo lamentable de las cientos de miles de víctimas mortales que se produjeron por el virus de la cepa SARS-CoV-2; sino también, por los desastres que éste ha dejado a su paso en todas las transversalidades espaciales (federales, estatales y municipales) y ámbitos longitudinales (diferentes grupos sociales) de la vida en sociedad, en todos y cada uno de los lugares del mundo conocido.

El fenómeno sanitario global por el que atraviesa la humanidad, ha dejado múltiples lecciones de vida a todos los pobladores, quizá una de las más evidentes sería que estas manifestaciones proclivemente negativas vinculadas a los procesos globalizantes, los cuales se han explorado, desde hace tiempo y desde diversas esferas teóricas, disciplinares y metodológicas, por diversos autores (Borja, J.: 2002; Ianni, O.: 2007; 2005; McLuhan, M. 2005; Sassen, 2007 y otros más); indicando unánimemente que esta manifestación de corte mundialista y sus expresiones globales; son capaces de transformar de facto en unos pocos meses, a toda la superestructura social construida por la colectividad urbana a través de los siglos. Es evidente que el mundo conocido ha entrado a una nueva realidad frágil, expuesta y vulnerable; ya que como menciona McLuhan: “... nos convertimos en una aldea global" (McLuhan, M. 2005:57).

Aún más, también estos procesos mundializantes han hecho entender a los gobernantes y a los gobernados, la fragilidad del equilibrio que existe entre la naturaleza originaria y la vida en la sociedad urbana; sobre todo en las grandes concentraciones metropolitanas donde la resiliencia y la sustentabilidad, como procesos operativos y estratégicos que debieran de alimentar a las políticas públicas y a la agenda de gobierno, tienden a representar la única forma para la recuperación de la ciudad, en una nueva realidad urbana que nos corresponde vivir en esta contemporaneidad líquida.

Incluso, es en estos tiempos donde conceptos propuestos anteriormente, por el autor de este escrito, en la esfera metropolitana de México y del sur global, como: la metrópoli prematura, las fuerzas centrifugas expansivas, fuerzas centripetas de cohesión poblacional, espacialidades no ciudad, contemporaneidad líquida y otros más ${ }^{2}$, adquieren mayor relevancia de aplicabilidad teórica, estratégica y metodológica, en el estudio y en la planeación espacial de estas grandes concentraciones de población.

Dichos conceptos involucrados en los procesos territoriales que permiten valorar el grado de consolidación espacial de las metrópolis, están enmarcados en la teoría que vincula, por un lado, a las fases por las que atraviesa lo urbano hacia lo metropolitano y por el otro, la determinación

\footnotetext{
${ }^{1}$ Nacionalidad: mexicano; Adscripción: Facultad de Arquitectura de la Universidad Autónoma de Nuevo León, México; doctor en Filosofía con Orientación en Arquitectura y Asuntos Urbanos; miembro del Sistema Nacional de Investigadores (CONACYT) ratificado en el Nivel 2; miembro de la Academia Mexicana de Ciencias AMC desde el año 2008; eduardo.sousagn@uanl.edu.mx

2 Para clarificar estos conceptos consultar Sousa-González: 2020; 2019; 2015; 2013, entre otros https://www.researchgate.net/profile/ Eduardo_Sousa-Gonzalez/research
} 
puntual que involucra a los ciclos del desarrollo urbano; estos ciclos mencionados son:

i. De concentración y primacia;

ii. De polarización regresiva; y

iii. De contraurbanización, según se

representan en la figura 1.

Figura 1. Ciclos del desarrollo urbano en el espacio metropolitano

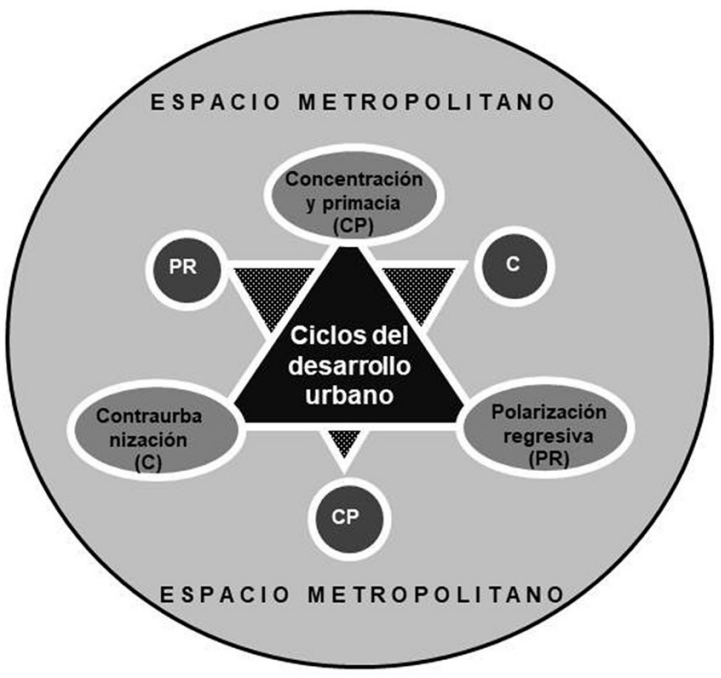

Fuente: Datos generados en esta investigación a partir de Sobrino, J. (2003)

Dichos ciclos aludidos tienden a enmarcar y clarificar el avance transformacional e iterativo, justamente, de la conurbación espacial metropolitana; enunasociedad conunacultura, una idiosincrasia y unos procesos identitarios urbanos distintivos y caracterizados por una entelequia adquirida evolutivamente por los ciudadanos; ya que la colectividad social lleva en su misma estructura social, histórica, contemporánea y líquida, aquel dinamismo intrínseco, que les hace en la cotidianeidad: producir, multiplicar y replicar las transformaciones espaciales evolutivas; las cuales tienden a desembocar en la generación de un dinamismo espacial-urbano periféricamente expansivo y galopante; el cual tiende a agregar físicamente cada vez más áreas conurbadas; modificando dinámicamente los linderos adyacentes del lugar.

Es en este estado de hechos por el que muchas de las metrópolis de México y del sur global, tienden a experimentar sus procesos evolutivos y de crecimiento espacial expansivo-periférico; adoleciendo, en muchos de los casos, de un modelo de planeación eficiente, efectivo y eficaz, que logre equilibrar su ensanchamiento espacial con: i. La infraestructura pertinente y necesaria; ii. La dotación de equipamientos imprescindibles; iii. La forma de producción de mercancías en los sectores secundario y terciario y; iiii. Los procesos de sustentabilidad del lugar. Ya que como menciona Harvey, D. “... la ciudad es creada por el hombre, pero también condenado a vivirla" (2019:19).

Es desde esta perspectiva que involucra a los espacios urbanos, a sus procesos de crecimiento expansivo, su arquitectura sus formas de diseño y sus múltiples variables intervinientes, que este número de CONTEXTO. Revista de la Facultad de Arquitectura de la Universidad Autónoma de Nuevo León, agrupa ocho trabajos de investigación de académicos investigadores de diferentes partes del mundo.

El primero es presentado por el doctor José Antonio Martínez Prades, quien es el Coordinador del Área de Historia del Arte del Departamento de Humanidades Contemporáneas de la Universidad de Alicante (España); dicho artículo es denominado "Antonio Serrano Peral y la construcción de la iglesia de nuestra señora de gracia en Alicante, España"; donde el autor se plantea indagar en las posibles fuentes de inspiración de Serrano Peral y en los procesos constructivos de la iglesia de Nuestra Señora de Gracia de Alicante, proyectada en 1945, a partir de una triple línea metodológica que trata datos biográficos, aspectos técnicos y condiciones contextuales de la posguerra española. El estudio se ha basado principalmente en la documentación existente en el Archivo General de la Administración del Estado y en el archivobiblioteca personal del propio autor.

El artículo de la doctora Maritza GranadosManjarrés, colombiana y Profesora Asistente en la Pontificia Universidad Javeriana de Colombia, lleva por nombre "Crear: especular y subvertir", mencionando que en éste se establece que, para hacer propuestas innovadoras en el proyecto arquitectónico, los diseñadores deben romper las leyes de lo que saben, ver más allá de lo que se da y subvertir el statu quo. La propuesta se sitúa en el cruce entre el ejercicio proyectual y los procesos cognitivos detrás de la creatividad, invitando a proyectar desde la ruptura que supone pensar universos aparentemente inconexos.

En el caso de las doctoras Nieves Martínez- 
Roldán y Lola Goytia-Goyenechea, adscritas a Escuela Técnica Superior de Arquitectura, Departamento de Urbanística y Ordenación del Territorio, de la Universidad de Sevilla, España; elaboran una investigación que titulan "Huella minera en la ciudad de Guanajuato (México) entre los siglos xvi - xix: morfología urbana y planimetría en el archivo de indias en Sevilla (España). En donde mencionan que la ciudad de Guanajuato es una ciudad singular: su localización, paisaje único e historia han condicionado la morfología urbanoterritorial de la ciudad y su entorno, evolucionando ambos junto a la minería y generando una interesante bibliografía y planimetría. Constituida por un centro histórico no muy longevo y una serie de asentamientos periféricos producto de la actividad minera, en Julio de 1982 la ciudad es declarada Zona de Monumentos Históricos por el Instituto de Antropología e Historia, en 1988 fue inscrita por la UNESCO en la Lista de Patrimonio Mundial bajo el nombre de Ciudad Histórica de Guanajuato y Minas Adyacentes, y desde 2006 tiene el reconocimiento de ser la Quinta Ciudad más Bella del Mundo, y todo ello gracias a su arquitectura y minería como recuerdo de su pasado colonial. El interés de este artículo radica en realizar un acercamiento a los elementos que han dejado su propia huella como consecuencia de la actividad minera, y referenciarlo a los planos que se encuentran localizados en el Archivo de Indias de Sevilla.

Las doctoras Vanessa Nagel Vega y Lourdes Cruz González Franco adscritas a la Facultad de Arquitectura de la Universidad Nacional Autónoma de México; presentan un artículo sobre la ciudad de Monterrey, titulado: "Voceando la modernidad. La renovación arquitectónica de Monterrey en el periódico El Norte (1945-1963)", el cual fue apoyado por el Programa de Becas Posdoctorales de la UNAM / DGAPA 2019; mencionando que la ciudad de Monterrey, Nuevo León, en el noreste de México, se caracteriza por un temprano desarrollo técnico que transformó la urbe y marcó su apropiación de la modernidad por el empleo de materiales locales industrializados de construcción. La difusión de las renovaciones arquitectónicas y urbanas que se iban sucediendo en las páginas del periódico El Norte, aportan una visión particular de la ciudad que nace industrial y que busca su conversión en el paradigma de la ciudad moderna occidental. Los edificios que se iban divulgando consolidarían dicho imaginario, al mismo tiempo que las carencias inherentes a las economías en crecimiento de los países latinoamericanos, marcan una crítica constante al proceso modernizador. La difusión en la prensa local, marcada por una fuerte inclinación hacia las obras de iniciativa privada, como la pionera construcción del campus del Instituto Tecnológico y de Estudios Superiores de Monterrey, iría dando cabida, paulatinamente, a las obras de presupuestos públicos, como aquellas significativas del Instituto Mexicano del Seguro Social y de la Universidad de Nuevo León. Así, el presente análisis busca ampliar y enriquecer las interpretaciones actuales sobre una más de las distintas modernidades nacionales.

En el caso del doctor Ramón Ramírez Ibarra, profesor-investigador de Facultad de Arquitectura de la Universidad Autónoma de Nuevo León, México, aborda un artículo titulado "Sociología de la movilidad: comprendiendo lo urbano desde la micrópolis"; donde expone que la movilidad es un tema central en el urbanismo del siglo XXI. Ha generado propuestas, acciones y políticas con importantes contribuciones para el desarrollo de la ciudad, pero, como objeto de estudio tiende a una reducción analítica en materia de ingeniería vial o arquitectura, motivada por una visión centrada en la morfología o el sitio, visión que refleja una comprensión parcial del fenómeno urbano, visto más como un proceso estático, depósito pasivo de planes, programas o normas de ejecución. En este trabajo, se propone una reflexión conceptual del concepto de movilidad en el espacio urbano, partiendo de una escala micrológica, es decir, desde la observación social en cuanto fenómeno particular y emergente del habitar humano como construcción cultural y tecnológica, tal como se propone en la sociología comprensiva. Este hecho, cualitativo, redunda en interés en la medida que el fenómeno de la movilidad es indisociable de todo proceso de autocomprensión del sujeto, en cuanto elemento de experiencia centrada y vinculante con una realidad cada vez más condicionada por factores de limitación, selectividad, densidad y adaptación para su desarrollo existencial.

El doctor Raúl Holguín Ávila profesorinvestigador del Instituto de Arquitectura Diseño y Arte de la Universidad Autónoma de Ciudad Juárez, propone una investigación sobre "La reivindicación del paisaje urbano histórico a través del espacio público: El centro de Ciudad Juárez como escenario simbólico de análisis"; 
mencionando que el interés del presente artículo es el de abordar la reivindicación del paisaje urbano histórico a través del espacio público y su relación con las prácticas y los imaginarios con la intención de establecer la importancia de incluir estos elementos al cuestionarse sobre las formas mediante las cuales se interviene, percibe y preserva el paisaje urbano histórico. Para ello, se desarrollaron 32 entrevistas en profundidad en dos tipologías de actores distintas relacionados con el centro de Ciudad Juárez, las cuales, mediante análisis de discurso, han permitido enfatizar en los aspectos tangibles e intangibles que dotan de significado el centro histórico puntualizando en el espacio público, las prácticas sociales y los imaginarios urbanos. Se menciona que entre los principales hallazgos, se destaca que la implementación de espacios públicos en la zona centro de la ciudad, en relación con la asignación de significados a determinados edificios patrimoniales, permite ampliar las dinámicas de interacción en dicho espacio, lo que resulta en la generación de lugares y prácticas que se orientan a la vinculación con el carácter patrimonial asignando valores positivos a las formas de percibir el centro y por lo tanto del paisaje urbano histórico.

En el caso del doctor Silverio Hernández Moreno profesor investigador de la Facultad de Arquitectura y Diseño de la Universidad Autónoma del Estado de México; profundiza en una temática sobre los "Requerimientos de diseño ambiental al interior de los edificios en México"; mencionando que el objetivo fue la revisión y análisis de la normatividad mexicana acerca de Calidad Ambiental al Interior de los Edificios que fuera equivalente a las normas estadounidenses que se solicitan durante el proceso de certificación LEED ${ }^{\circledR}$ v4; con el propósito de certificar edificios en México. La metodología se resume en la revisión de normas mexicanas que tuvieran equivalencia con la estadounidense y en concordancia con sus contenidos, su rigor de aplicación y su vigencia. Como resultado, se obtuvo que en la mayoría de los requerimientos solicitados por la categoría de Calidad Ambiental al Interior de las Edificaciones no hubo equivalencia entre normas, y por tanto se concluye, en lo general, que mientras las normas mexicanas no sean lo suficientemente completas para aplicación en este tipo de modelos o sistemas de clasificación ambiental en edificios, se seguirá cumpliendo con las normas estadounidenses o de ISO siempre y cuando tengan una adecuada adaptación al contexto mexicano por parte de los proponentes".

La doctora María Adriana Gebauer Muñoz profesora-investigadora de la Facultad de Arquitectura y Diseño de la Universidad de la Salle en Pachuca estado de Hidalgo, México, aborda el tema "Borde costero ciudad Antofagasta, Chile, visto como interfase glocal "; mencionando que el objetivo de este artículo es, por una parte, presentar y proponer los lugares, que en el bordecostero de Antofagasta se pueden desarrollar como interfase entre lo global y lo local, a partir de considerar análisis y conclusiones de cuatro dimensiones de abordaje de la Tesis Doctoral: "Centro Histórico como interfase glocal" para la inserción de la ciudad intermedia en el sistema global de ciudades: Caso de estudio Antofagasta, Chile, 1990/2012" (Gebauer, 2015), considerando la importancia que, en esta ciudad, adquiere este borde cuando actúa y se configura como espacio mediador transnacional, y por otra, incorporar los conceptos de interfase glocal, Ciudad Intermedia, $\mathrm{CH}$-ampliado, y ciudad-región ampliada, que fueron definidos por dicha Tesis.

Por último, en la publicación de la Revista se incluye una Reseña del doctor Eduardo Sousa-González; del libro titulado "El espacio metropolitano contemporáneo. Desde las políticas públicas hasta el modelo de planeación"; editado por la editorial Colofón en el año 2020.

Como comentario final, los miembros del equipo que conformanaCONTEXTO.RevistadelaFacultad de Arquitectura de la Universidad Autónoma de Nuevo León, estamos plenamente convencidos que el investigador y el lector común interesado en la diversa temática expuesta, encontraran a través de las páginas que componen este proyecto editorial, un tópico con una visión interdisciplinar, de actualidad e internacional; enfocada en diferentes esferas del quehacer académico-investigativo, en donde, investigadores de alta calificación, han colaborado con sus trabajos personales o grupales en la generación de este número.

Seguramente los artículos que se incluyen aquí serán no solamente de interés, sino también de utilidad concreta en futuras investigaciones, complaciendo al investigador y al lector más exigente.

Finalmente, es necesario reconocer que lo expuesto a través de este número de CONTEXTO, es un esfuerzo continuado a lo largo de muchos 
años por mantener una calidad de excelencia editorial, que ha sido compartido por autores, pares de evaluadores, el Comité Editorial, la Coordinación Editorial, el equipo de apoyo y por supuesto, las autoridades institucionales que forman la Facultad de Arquitectura, su Posgrado y la Universidad Autónoma de Nuevo León. C

\section{REFERENCIAS BIBLIOGRÁFICAS}

Borja, J. (2002). Local y global. La gestión de las ciudades en la era de la información. México; Taurus

HARVEY, D. (2019). Ciudades rebeldes. Del derecho a la ciudad a la revolución urbana

Ianni, O. (2007). La sociedad global. México Siglo $\mathrm{XXI}$

Ianni, O. (2005). La era del globalismo. México Siglo XXI

Ma. Luhan; et al. (2005). La aldea global. Barcelona, España; Gedisa

Sassen, S. Los espectros de la globalización. México FCE

Sobrino, J. (2003). Competitividad de las ciudades de México; El colegio de México.

Sousa-González, E.: (2020). El espacio metropolitano contemporáneo. Desde las políticas públicas hasta el modelo de planeación. México; COLOFÓN

Sousa-González, E.: (2019). El espacio interior de la ciudad metropolitana. México; COLOFÓN

Sousa-González, E.: (2015). De la ciudad a la metrópoli. Nuevas realidades territoriales. Chile; Trama impresores

Sousa-González, E. et al: (2013). Espacio urbano reconstrucción y reconfiguración territorial. Chile; Trama impresores 